Sachs disease (a G-gangliosidosis with deficiency of B-hexosaminidase A), cerebrotendinous xanthomatosis (deficiency of mitochondrial enzyme sterol 27-hydroxylase involved with bile-acid synthesis), DNA polymerase g disorder (mitochondrial recessive ataxia syndrome), and spinocerebellar ataxia with axonal neuropathy. Early-onset ataxia with cerebellar atrophy: ataxia telangiectasia; ataxia with oculomotor apraxia types 1 and 2, caused by mutations of the aprataxin and senataxin genes; ataxia of Charlevoix-Saquenay (sacsin gene); infantile-onset spinocerebellar ataxia (a gene encoding the proteins twinkle and twinky); Cayman ataxia (gene encoding caytaxin); and Marinesco-Sjogren syndrome (rare infantileor childhood-onset ataxia, with cataracts, mental retardation, myopathy, hypogonadotropic hypogonadism, and skeletal deformities). Friedreich ataxia is the most prevalent, and directed genetic testing is recommended based on clinical phenotype, which avoids the expense of an unfocussed molecular diagnostic battery. (Fogel BL, Perlman S. Clinical features and molecular genetics of autosomal recessive cerebellar ataxias. Lancet Neurol March 2007;6:245-257). (Respond: Dr Susan Perlman, UCLA Ataxia Center, University of California at Los Angeles, 710 Westwood Plaza, Los Angeles, CA 90095).

COMMENT. Hereditary ataxias are autosomal dominant, autosomal recessive, Xlinked, and mitochondrial. The clinical phenotype is important in the differentiation of these ataxias, especially when a characteristic family history is unavailable. Neuroimaging is also useful to differentiate ataxias with and without cerebellar atrophy.

Cerebellar ataxia in Norway. Among 60 individuals from 39 families with ataxia, age at onset was lower for patients with autosomal recessive (ARCA) compared with autosomal dominant cerebellar ataxias (ADCA). Disease prevalence in Oslo is estimated at 2.2/100,000 for ARCA and 3.0/100,000 for ADCA. Surprisingly, no Norwegian family with Friedreich ataxia was found in the patients referred to a Department of Neurology in Oslo. Pediatric patients may have been treated elsewhere. ((Koht J, Tallaksen CME. Acta Neurol Scand May 2007;115(Suppl 187):76-79).

\title{
BETAMETHASONE TREATMENT OF ATAXIA-TELANGIECTASIA
}

The response to corticosteroid therapy in a 3-year-old boy with ataxia-telangiectasia is reported from University of Siena, Italy. The diagnosis was confirmed by molecular testing. Improvements in neurologic signs were noted by the parents when betamethasone was used occasionally to treat asthmatic bronchitis. After 2 or 3 days continuous therapy with betamethasone, $0.1 \mathrm{mg} / \mathrm{kg} / 24 \mathrm{hrs}$, divided every 12 hours, a beneficial effect was observed. The improvement in stance, gait, and skilled movements was dramatic after 2 weeks of treatment, but adverse effects including increased appetite and body weight and moon face occurred by 4 weeks. Methylprednisolone, $2 \mathrm{mg} / \mathrm{kg} / 24 \mathrm{hrs}$, divided every $12 \mathrm{hrs}$, substituted at 4 weeks, was ineffective, and was discontinued. At 6 months follow-up, without therapy, the child showed severe signs of CNS impairment. (Buoni S, Zannolli R, Sorrentino L, Fois A. Betamethasone and improvement of neurological symptoms in ataxia-telangiectasia. Arch Neurol 2006;63:1479-1482). (Respond: Raffaella Zannolli MD, Department of Pediatrics, Policlinico Le Scotte, University of Siena, Siena, Italy). 
COMMENT. Despite the potential adverse effects of corticosteroids in a patient already immunocompromised, a trial of betamethasone was considered appropriate in view of the poor neurologic prognosis in ataxia-telangiectasia. Limited trials of gabapentin, pregabalin and tiagabine were moderately effective in the control of ataxia in an adult with ataxia-telangiectasia (Gazulla $\mathbf{J}$ et al. New therapies for ataxia-telangiectasia. Arch Neurol April 2007;64:607-609).

\section{HEADACHE DISORDERS}

\section{FAMILIAL HEMIPLEGIC MIGRAINE WITH ATP1A2 MUTATIONS}

Three children with prolonged hemiplegia following severe unilateral headache and having mutations in ATP1A2 are reported from UCLA School of Medicine, Los Angeles, CA; University Children's Hospital, Zurich, Switzerland; and Wake Forest University School of Medicine, Winston-Salem, NC. Patient 1, a 9-year-old girl developed left-sided numbness and paralysis after 2 days severe right-sided headache. The hemiparesis lasted 5 days and then gradually resolved. Brain MRI and angiography and lumbar puncture were normal. EEG showed slowing in right hemisphere. No previous attacks were reported in the child, but her mother had a history of episodic hemiparesis lasting $30 \mathrm{~min}$ to an hour, and accompanied by headache and vomiting, since age 32 years. Patient 2, a 7-year-old boy had headache, somnolence, confusion and left hemiplegia, associated with viral gastroenteritis. Lumbar puncture and initial MRI were normal. Repeat MRI 2 days later showed increased signal intensity on diffusion weighted imaging in right parietal region. Hemiplegia persisted 7 days and gradually resolved over weeks. A generalized tonic-clonic seizure occurred 6 days after onset of hemiplegia. The child had a prior history of typical hemiplegic migraine, lasting 30 min and followed by unilateral headache, often induced by minor head trauma. Family history was negative. Patient 3 , a 10-year-old girl developed a headache a few hours after striking her head in a fall while skating. She did not lose consciousness. Two-3 hours later, she developed right-sided numbness, hemiplegia and global aphasia. Symptoms persisted 10 days, the weakness resolved in 3 weeks, and language returned to normal after 7 weeks. Brain MRI on days 2 and 10 were normal. EEG showed slowing on the left that persisted several weeks. Her father had a history of typical hemiplegic migraine beginning at age 7 years; also, episodic visual aura without headache, and a single seizure.

Three distinct ATP1A2 mutations were identified in the FHM2 gene, but none in the FHM1 gene of the patients. Affected parents of patients 1 and 3 also had the mutation. In Patient 2, neither parent had the mutation, indicating that the mutation in the child arose de novo. (Jen JC, Klein A, Boltshauser E et al. Prolonged hemiplegic episodes in children due to mutations in ATP1A2. J Neurol Neurosurg Psychiatry May 2007;78:523-526). (Respond: Dr Joanna C Jen, UCLA Neurology, 710 Westwood Plaza, Los Angeles, CA 90095).

COMMENT. Episodes of hemiplegic migraine usually last from 30 to $60 \mathrm{~min}$, but patients with more prolonged hemiparesis are reported. FHM1 is typically associated with cerebellar findings, and FHM2 with seizures. More than 20 mutations in ATP1A2 have been found to cause FHM2. The MRI is generally normal, but the EEG may show slowing over the affected hemisphere. 